\title{
An analysis of baseline data from the PROUD study: an open-label randomised trial of pre-exposure prophylaxis
}

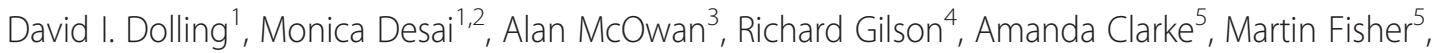
Gabriel Schembri ${ }^{6}$, Ann K. Sullivan ${ }^{3}$, Nicola Mackie”, lain Reeves ${ }^{8}$, Mags Portman ${ }^{9}$, John Saunders ${ }^{9}$, Julie Fox ${ }^{10}$, Jake Bayley ${ }^{11}$, Michael Brady ${ }^{11}$, Christine Bowman ${ }^{12}$, Charles J. Lacey ${ }^{13}$, Stephen Taylor ${ }^{14}$, David White ${ }^{14}$, Simone Antonucci ${ }^{3}$, Mitzy Gafos ${ }^{1}$, Sheena McCormack ${ }^{*}$, Owen N. Gill ${ }^{2}$, David T. Dunn ${ }^{1}$, and Anthony Nardone ${ }^{2}$ On behalf of the PROUD Study Group

\begin{abstract}
Background: Pre-exposure prophylaxis (PrEP) has proven biological efficacy to reduce the sexual acquisition of the human immunodeficiency virus (HIV). The PROUD study found that PrEP conferred higher protection than in placebo-controlled trials, reducing HIV incidence by $86 \%$ in a population with seven-fold higher HIV incidence than expected. We present the baseline characteristics of the PROUD study population and place the findings in the context of national sexual health clinic data.
\end{abstract}

Methods: The PROUD study was designed to explore the real-world effectiveness of PrEP (tenofovir-emtricitabine) by randomising HIV-negative gay and other men who have sex with men (GMSM) to receive open-label PrEP immediately or after a deferral period of 12 months. At enrolment, participants self-completed two baseline questionnaires collecting information on demographics, sexual behaviour and lifestyle in the last 30 and 90 days. These data were compared to data from HIV-negative GMSM attending sexual health clinics in 2013, collated by Public Health England using the genitourinary medicine clinic activity database (GUMCAD).

Results: The median age of participants was 35 (IQR: 29-43). Typically participants were white (81\%), educated at a university level (61 \%) and in full-time employment (72\%). Of all participants, 217 (40\%) were born outside the UK. A sexually transmitted infection (STI) was reported to have been diagnosed in the previous 12 months in 330/515 (64 \%) and 473/544 (87 \%) participants reported ever having being diagnosed with an STI. At enrolment, 47/280 (17\%) participants were diagnosed with an STI. Participants reported a median (IQR) of 10 (5-20) partners in the last 90 days, a median (IQR) of $2(1-5)$ were condomless sex acts where the participant was receptive and $2(1-6)$ were condomless where the participant was insertive. Post-exposure prophylaxis had been prescribed to 184 (34\%) participants in the past 12 months. The number of STI diagnoses was high compared to those reported in GUMCAD attendees.

Conclusions: The PROUD study population are at substantially higher risk of acquiring HIV infection sexually than the overall population of GMSM attending sexual health clinics in England. These findings contribute to explaining the extraordinary HIV incidence rate during follow-up and demonstrate that, despite broad eligibility criteria, the population interested in PrEP was highly selective.

Trial registration: Current Controlled TrialsISRCTN94465371. Date of registration: 28 February 2013.

Keywords: Pre-exposure prophylaxis (PrEP), Men who have sex with men (MSM), HIV prevention, Tenofovir, Truvada

\footnotetext{
* Correspondence: s.mccormack@ucl.ac.uk

${ }^{1}$ MRC Clinical Trials Unit at UCL, Aviation House, 125 Kingsway, London WC2B 6NH, UK

Full list of author information is available at the end of the article
} 


\section{Background}

Pre-exposure prophylaxis (PrEP) has proven biological efficacy to reduce the sexual acquisition of human immunodeficiency virus (HIV). In 2012 the US Food and Drugs Administration approved the use of daily Truvada (tenofovir and emtricitabine) as PrEP [1] based on several placebo-controlled randomised trials [2-4].

In England, gay and other men who have sex with men (GMSM) are the population most likely to acquire HIV sexually, according to surveillance data routinely collected from sexual health clinics. Using the CD4 back calculation estimate of HIV incidence, 2470 men who have sex with men (MSM) were estimated to have acquired their HIV infection in the UK in 2013. This number has increased steadily from 2004 and now accounts for $51 \%$ of all new HIV infections [5].

In addition to its biological benefits, the clinical delivery of PrEP currently requires contact with clinical services to enable regular testing for HIV and sexually transmitted infections (STIs), as well as access to behavioural interventions. Modelling suggests that introducing PrEP would have a large impact on the UK epidemic [6], in the context of provision to an appropriate population [7].

In England, sexual health for GMSM at risk of HIV is primarily provided by a network of over 200 open-access free clinics with 92,037 HIV-negative GMSM attending in 2013. To roll out PrEP in this setting, evidence of effectiveness that takes account of any change in risk behaviour is required [8]. This could only be captured in an open-label trial without a placebo control, ideally within a schedule and in a setting where PrEP could be delivered realistically (in terms of capacity and resources) [9]. Factors that could limit the population effectiveness of PrEP include changes in sexual behaviour [10] and the emergence of drug resistance [11]. In a nationally funded health service, it is also essential to consider the potential impact of funding diversion on other prevention activities and delivery of clinical services $[12,13]$.

The PROUD study demonstrated that the inclusion of PrEP gave a relative reduction of $86 \%$ in the incidence of HIV, with no infections among participants taking
PrEP, and refuted concerns that real-world effectiveness would be compromised [14]. HIV incidence in the study population was shown to be approximately seven times higher than the estimate for GMSM attending sexual health clinics in 2012. Here we present the baseline characteristics of the PROUD study population and place the findings in the context of national data returned from the sexual health clinics.

\section{Methods}

The PROUD trial was designed to explore the real-world effectiveness of PrEP in which eligible HIV-negative GMSM received open-label tenofovir-emtricitabine (TDFFTC) either immediately, in a risk reduction package, or after a deferral period of 12 months follow-up. The intention was of implementing this in sexual health clinics and with study procedures as close as possible to routine care in this setting. This paper reports the characteristics of participants recruited during a pilot phase, which aimed to establish the feasibility of a complete trial. However, the unexpectedly large number of HIV infections during the deferral period led to a recommendation from the Trial Steering Committee in October 2014 that all participants be offered PrEP. The findings on the effectiveness of PrEP were published and a larger trial is no longer required [14]. Trial procedures during the study are described in detail elsewhere [14].

The anticipated target for an adequately powered trial was 5000 (2500 per arm). This was based on an estimated incidence of 3/100 person-years during the first year in participants who were waiting to access Truvada and a $50 \%$ reduction in incidence in those offered Truvada. An arbitrary $10 \%$ target of 500 for the pilot phase was a pragmatic choice to guide as to whether 5000 participants could be enrolled over 2 years, according to the eligibility criteria in Table 1.

Potentially eligible GMSM were identified during routine attendances at 13 sexual health clinics in England, 8 in London and 5 outside (Birmingham, Brighton, Manchester, Sheffield and York). Participants with

Table 1 Eligibility requirements

\begin{tabular}{|c|c|}
\hline Inclusion criteria & Exclusion criteria \\
\hline $\begin{array}{l}\text { 1. Born to male gender, age } 18 \text { years or older } \\
\text { 2. Previously attended the enrolling clinic on at least one occasion } \\
\text { 3. Completed a screen for HIV and STIs } \\
\text { 4. HIV-negative by a routinely used assay within } \\
4 \text { weeks prior to or on the day of randomisation } \\
\text { 5. Reported unprotected anal intercourse (UAI) on more than } \\
\text { one occasion within the } 90 \text { days prior to randomisation } \\
\text { 6. Likely, in the opinion of the volunteer, to have UAI in the next } 90 \text { days } \\
\text { 7. Willing and able to comply with the visit schedule throughout the } \\
\text { follow-up period } \\
\text { 8. Willing and able to provide written informed consent }\end{array}$ & $\begin{array}{l}\text { 1. An acute viral illness that could be due to HIV seroconversion } \\
\text { 2. Any contraindications to Truvada according to the current } \\
\text { package insert } \\
\text { 3. Treatment for hepatitis B infection indicated or ongoing } \\
\text { 4. Unlikely, in the opinion of the clinician, to comply with the } \\
\text { randomised allocation }\end{array}$ \\
\hline
\end{tabular}

HIV human immunodeficiency virus, STI sexually transmitted infection 
regular sexual partners (in the opinion of the potential volunteer) who also met eligibility requirements were encouraged to enrol at the same time and both partners were randomly allocated to the same trial arm, minimising the possibility of PrEP being shared. Posters and electronic screens in participating sexual health clinics, as well as advertisements on social media, helped to promote the study. Business cards and leaflets advertising the study were also handed out by community organisations during outreach activities, including efforts to raise awareness of PrEP amongst GMSM. There was no financial payment for participants joining the study, nor were travel costs or other expenses paid for.

A screening visit was not required as the eligibility data on HIV are collected routinely. The Participant Information Sheet was shared with volunteers prior to enrolment. The research team at the clinic determined eligibility through a structured discussion with the volunteer and written informed consent was collected prior to enrolment. Eligible participants were randomised using a web-based tool incorporated within the database at each clinic. At the enrolment visit participants were asked to self-complete, in private, two paper baseline questionnaires (Additional files 1 and 2) on separate booklets collecting information on: demographics, sexual behaviour and lifestyle in the last 30 and 90 days; perception of HIV risk at the last condomless sex act; risk management strategies; past history of STIs; drug and alcohol use; depression severity captured by the Patient Health Questionnaire-9 (PHQ-9) [15]; motivation for taking part in the study; and perceptions regarding adherence to taking a daily pill. The questionnaires were derived from other studies in MSM populations and were being tested as part of the pilot phase. Questionnaires took less than 30 minutes to complete and were placed in a sealable envelope and sent to the Medical Research Council Clinical Trials Unit (MRC CTU) at University College London (UCL) for data entry without clinic staff seeing the responses.

A screen for STIs was performed, if indicated, according to routine clinical practice. A sample for antibody/antigen HIV testing was collected on the day of enrolment (Table 1).

In NHS sexual health clinics, basic demographic, diagnostic and service data are also returned for each attendance and collated by Public Health England, using the genitourinary medicine clinic activity database version 2 (GUMCADv2). GUMCADv2 is a pseudo-anonymised patient-level electronic dataset collecting information on diagnoses made and services provided by genitourinary medicine (GUM) clinics (level 3) and other commissioned level 2 (non-GUM) sexual health services [16]. For this analysis, data were extracted from GUMCADv2 on GMSM who were HIV-negative or of unknown status from all clinics in England between January 2013 and December 2013. Data were extracted on key demographics including age, ethnicity and place of birth, STI diagnoses by number of GMSM, as opposed to by GMSM attendances, (pharyngeal, urethral and rectal chlamydia and gonorrhoea, primary secondary and early latent syphilis, hepatitis B and C infections, lymphogranuloma venereum (LGV)), HIV tests (number of HIV tests and average number per GMSM), and episodes of postexposure HIV prophylaxis (PEP) among GMSM.

The data were analysed using Stata 13.1 [17]. Comparisons of categorical data were conducted using a $\chi^{2}$ test or a two-tailed Fisher exact test where numbers were less than five in any group. Participants recruited within London were compared to those recruited outside of London to determine differences in baseline demographics, sexual behaviour and lifestyle. Continuous variables were compared using a Mann-Whitney $U$ test. Multiple component analysis (MCA) was used to determine whether responses to questions where participants could select multiple answers decomposed into distinct groups [18].

The PROUD study protocol was approved by London Bridge Research Ethics Committee, the Medicines and Healthcare products Regulatory Agency and each participating hospital trust (Chelsea and Westminster Healthcare NHS Foundation Trust, London, UK; Brighton and Sussex University Hospitals NHS Trust, Brighton, UK; Homerton University Hospital NHS Foundation Trust, London, UK; Central Manchester University Hospitals NHS Foundation Trust, Manchester, UK; Imperial College Healthcare NHS Foundation Trust, London, UK; Sheffield Teaching Hospitals NHS Foundation Trust, Sheffield, UK; York Teaching Hospital and Hull York Medical School, York, UK; Barts Health NHS Trust, London, UK; King's College Hospital NHS Foundation Trust, London, UK; Guy's and St Thomas' NHS Foundation Trust, London, UK; and Heart of England NHS Foundation Trust, Birmingham, UK).

\section{Results}

\section{Recruitment}

The PROUD pilot study enrolled the first participant on 27 November 2012. The pilot study was initially expected to fully recruit within 6 months, but this target was modified in April 2013 due to slow recruitment, attributed to lack of study resources delaying clinic activation, and low awareness of PrEP in the community [19]. The modified target was that full recruitment would be reached in April 2014. The two cumulative targets and actual recruitment are shown in Fig. 1. Recruitment was stopped at the end of April 2014 after 544 participants were enrolled. Two participants enrolled twice to access PrEP and were analysed in their original trial arm, the deferred group (Fig. 2). In total, 19 participants enrolled 


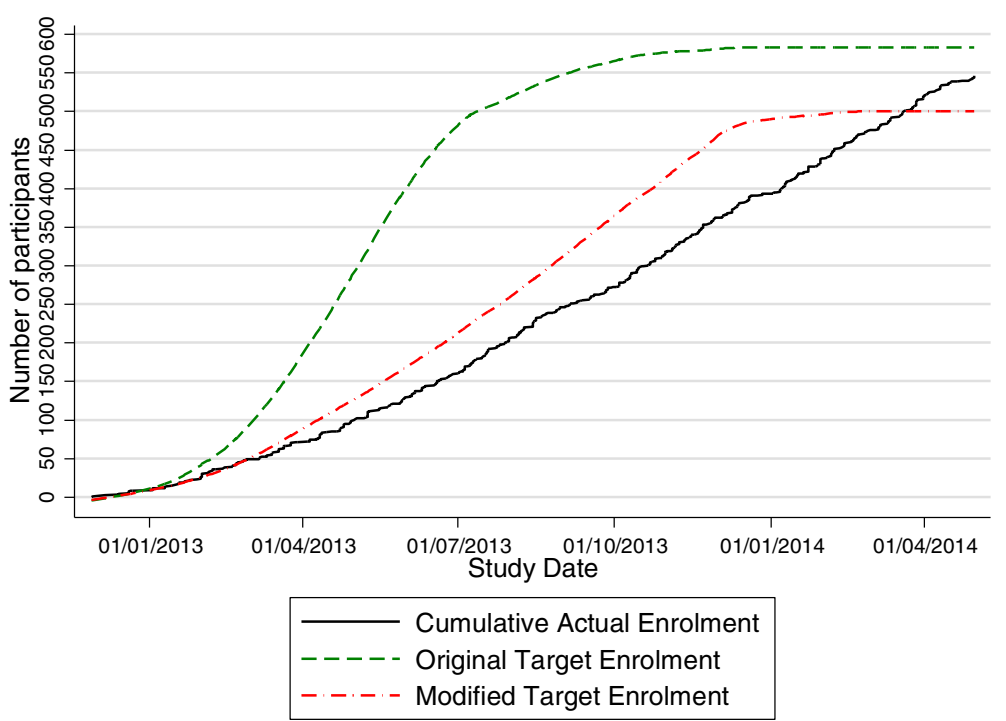

Fig. 1 Cumulative recruitment by time

with their partner in the trial and were randomised to the same trial arm.

\section{Baseline demographics}

Data from the self-completed questionnaire were available for 540/544 participants (99\%); missing questionnaires were either lost $(n=3)$ or not completed by participants $(n=1)$.
Baseline demographic data are shown in Table 2. The median age of participants was 35 (interquartile range (IQR): 29-43) with an age range of $18-75$, and the majority of participants were of white ethnicity (81\%). Participants who described themselves as of an 'Other' ethnicity defined themselves as Chinese $(n=11)$, Latin American $(n=10)$, Arabic $(n=5)$, Asian $(n=5)$ or mixed race or other $(n=5)$.

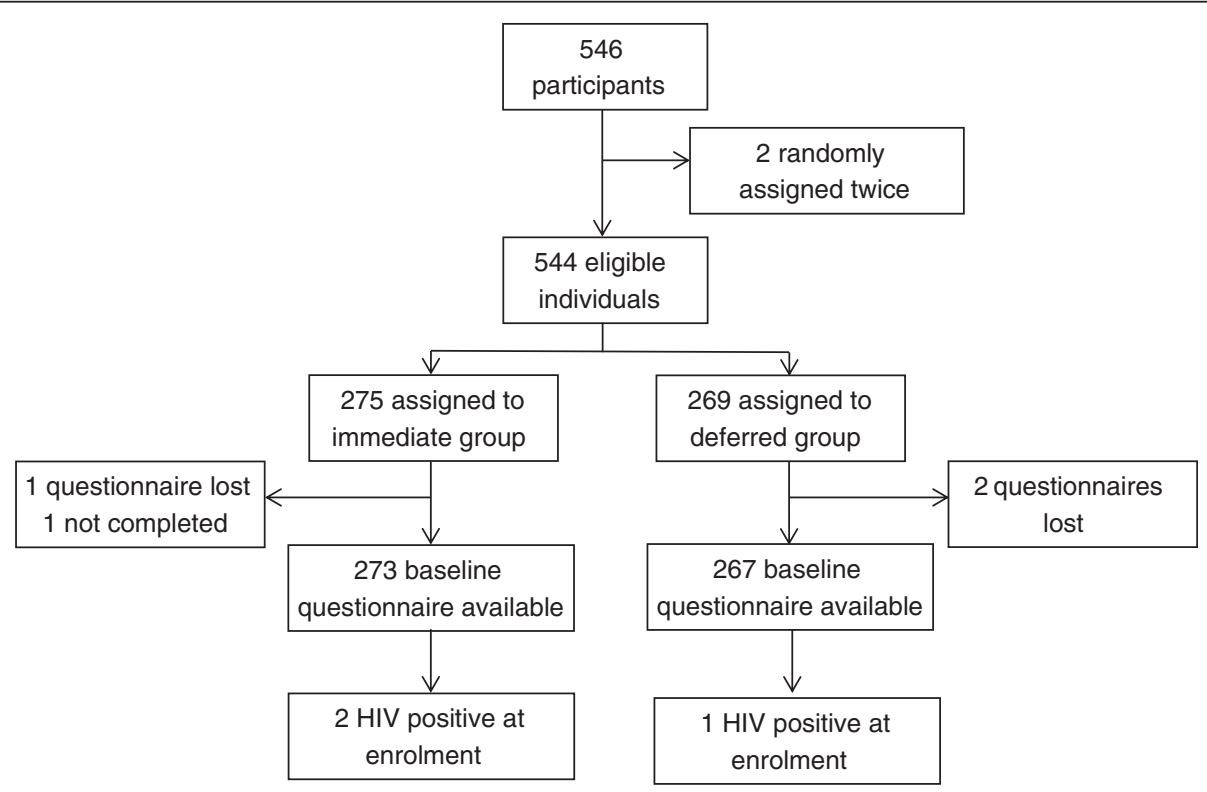

Fig. 2 Flow diagram of enrolment 
Table 2 Baseline demographic data

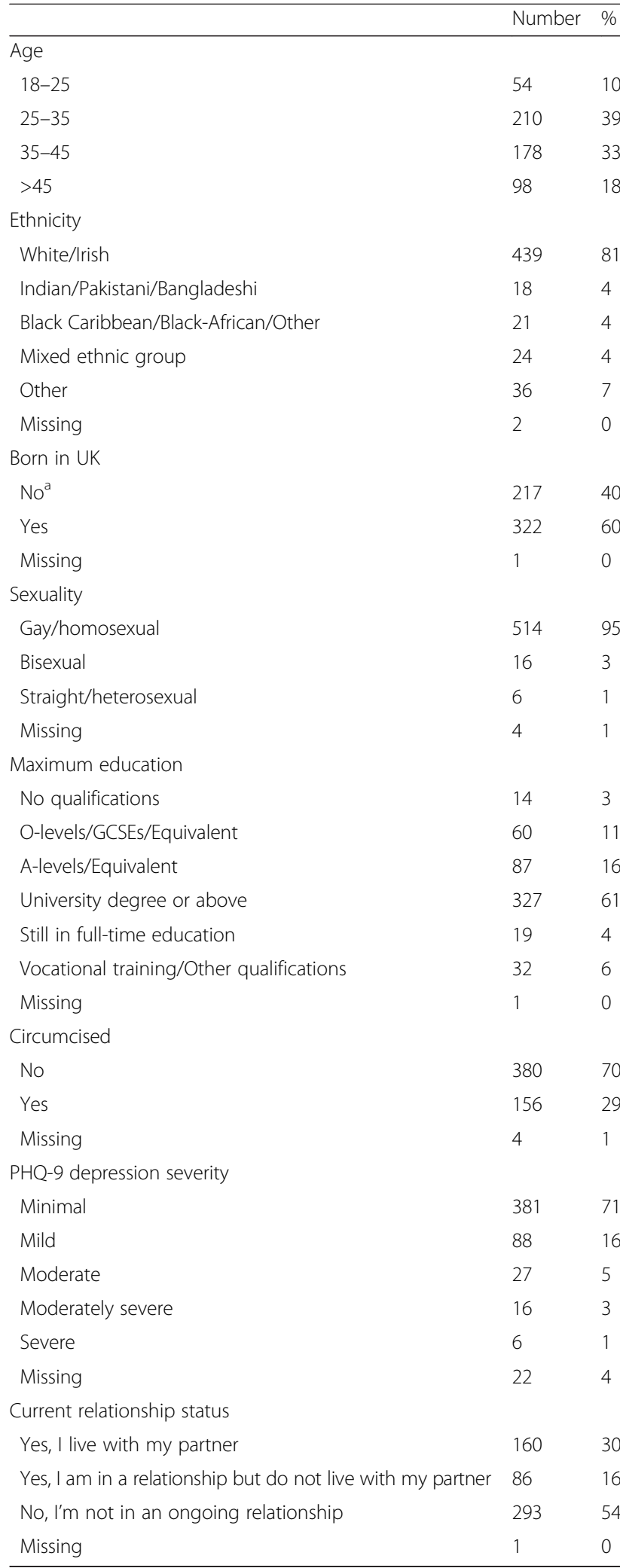

37\% Europe, 13 \% Africa, 12 \% Central/South America, $12 \%$ Asia, $12 \%$ North America, $10 \%$ Australasia and $4 \%$ missing
Typically participants were highly educated, $61 \%$ were educated at a university degree level or above. Participants were predominantly employed either full-time $(72 \%)$ or part-time (10\%). Of all participants, 217 (40\%) were born outside the UK. In total, 156 (29 \%) participants were circumcised and circumcision was significantly higher in participants born outside the UK $(n=84 ; 39 \%$ versus $n=72$; $23 \% ; p<0.001)$. The majority of participants described their sexuality as gay/homosexual $(n=514 ; 95 \%), 16(3 \%)$ as bisexual and $6(1 \%)$ as straight/heterosexual. Only one participant defined themselves as transgender. A prevalence rate for moderate to high severity of depressive symptoms of $9.5 \%$ was identified, lower than those observed in HIV-1-positive MSM in the Antiretrovirals, Sexual Transmission Risk and Attitudes (ASTRA) study $(27.0 \%)$ [20]. The prevalence of a major depressive disorder was $5.0 \%$, higher than the $2.5 \%$ observed in a general European male population [21]. Almost half (46\%) of the participants described themselves as being in an ongoing relationship and 160 (30\%) were currently living with their partner.

\section{Sexually transmitted infections}

STI data from the clinic interview (self-reported lifetime diagnoses), self-completed questionnaire (self-reported diagnoses in the last 12 months) and baseline STI test are shown in Table 3. Self-reported data on STI history were available for 515/544 (94\%) participants but the exact denominators differed by STI. A diagnosis of any STI in a participant's lifetime was reported in $473 / 544$ $(87 \%)$ participants. This was frequently urethral gonorrhoea $(n=216 ; 40 \%)$, oral gonorrhoea $(n=175 ; 32 \%)$, rectal gonorrhoea $(n=174 ; 33 \%)$, urethral chlamydia $(n=173 ; 32 \%)$ and genital warts $(n=168 ; 31 \%)$. Participants reported being screened for STIs a median (IQR) of $3(2-4)$ times in the previous 12 months. One or more STI diagnoses in the previous 12 months was reported in 330/515 (64\%) participants, in particular: rectal gonorrhoea $(n=126 ; 26 \%)$, oral gonorrhoea $(n=121$; $25 \%)$, urethral gonorrhoea $(n=112 ; 24 \%)$ and rectal chlamydia $(n=99 ; 21 \%)$. At enrolment 47/280 (17\%) participants screened, based on clinical indication, were diagnosed with an STI. These were mostly oral gonorrhoea $(n=13)$, syphilis $(n=13)$, rectal gonorrhoea $(n=12)$ and rectal chlamydia $(n=10)$ diagnoses. In addition, three participants were found to be HIV-1-positive despite having an HIV-negative test in the 4 weeks prior to randomisation. These participants are included in these analyses of baseline data.

\section{Reported sexual risk management strategies and risk perception}

Participants had attended a clinic a median (IQR) of 3 (2-4) times in the past 12 months for an HIV test. A 
Table 3 Self-reported history of, and diagnosed sexually transmitted infections at enrolment

\begin{tabular}{|c|c|c|c|c|c|c|}
\hline & \multicolumn{2}{|c|}{ Self-reported diagnoses (lifetime) ${ }^{a}$} & \multicolumn{2}{|c|}{ Self-reported diagnoses (last 12 months) ${ }^{b}$} & \multicolumn{2}{|c|}{ Diagnosed at enrolment } \\
\hline & $N$ & $\%$ & $N$ & $\%$ & $N$ & $\%$ \\
\hline Rectal gonorrhoea & $174 / 532$ & 33 & $126 / 478$ & 26 & $12 / 251$ & 5 \\
\hline Urethral gonorrhoea & $216 / 538$ & 40 & $112 / 480$ & 24 & $2 / 256$ & 1 \\
\hline Oral gonorrhoea & $175 / 539$ & 32 & $121 / 483$ & 25 & $13 / 255$ & 5 \\
\hline Rectal chlamydia & $160 / 533$ & 30 & $99 / 470$ & 21 & $10 / 248$ & 4 \\
\hline Urethral chlamydia & $173 / 538$ & 32 & $80 / 478$ & 17 & $3 / 255$ & 1 \\
\hline Oral chlamydia & $63 / 523$ & 12 & $60 / 471$ & 13 & $3 / 244$ & 1 \\
\hline Lymphogranuloma venereum (LGV) & $15 / 127$ & 12 & $10 / 458$ & 2 & $0 / 7$ & 0 \\
\hline Syphilis & $110 / 537$ & 20 & $49 / 473$ & 10 & $13 / 237$ & 5 \\
\hline Hepatitis C & $9 / 451$ & 2 & $3 / 464$ & 1 & $0 / 132$ & 0 \\
\hline Genital warts & 168 & - & $45 / 472$ & 10 & 6 & - \\
\hline Genital herpes & 73 & - & $25 / 464$ & 5 & 10 & - \\
\hline
\end{tabular}

Notes

athe data for lifetime diagnoses were collected by staff during the enrolment interview and accounted for conditions that were never tested for

b the data for diagnoses in the last 12 months were reported by participants on the questionnaire who were invited to indicate 'yes' or 'no' for each infection. In the event that no answer was returned, they were not included in the denominator for that infection

course of PEP had been prescribed to 184 (34 \%) participants in the past 12 months with $71(13 \%)$ having more than one course.

Overall $50 \%$ of participants reported more than one strategy for managing their risk of contracting HIV. The following strategies were reported: using condoms for anal sex $(n=209 ; 39 \%)$; choosing partners based on their negative HIV status $(n=199 ; 37 \%)$; strategic positioning (being 'on top' if they are unsure of partner's HIV status) ( $n=157 ; 29 \%)$; asking partners to use a condom for anal sex $(n=134 ; 25 \%)$; seeking partners who are known to be on HIV treatment $(n=134 ; 25 \%)$. Only 75 (14\%) participants reported that they did not think about risk reduction strategies. MCA highlighted two clear groups: 113 participants who exclusively reported condom use (self or partner) and 206 who reported serosorting or strategic positioning strategies. The other 131 participants used a combination of these strategies.

Participants were asked to self-categorise, into one of five groups, their perceived risk of contracting HIV if they have anal sex without using a condom: $8(2 \%)$ described themselves as at no risk; $146(27 \%)$ at little risk; $258(49 \%)$ as somewhat at risk; $85(16 \%)$ as at high risk; and $34(6 \%)$ at very high risk. Participants who considered themselves somewhat at risk or higher $(n=377)$ were more likely to have been diagnosed with syphilis, hepatitis $C$, rectal gonorrhoea or rectal chlamydia in the past 12 months $(n=151 ; 40 \%)$ than those who considered themselves at little risk or lower $(n=46 ; 28 \% ; p=0.009)$.

\section{Sexual risk behaviour}

In the last 90 days, participants reported a median (IQR) of $10(5-20)$ partners with a range of 1 to $>100$. Of these, a median (IQR) of $2(1-5)$ were condomless partners where the participant was receptive (bottom), and a median (IQR) of 2 (1-6) were condomless partners where the participant was insertive (top). A median (IQR) of 7 $(2-15)$ partners were new (partners a participant had not had sex with before). In the last 90 days, 55 (10 \%) participants had 40 or more partners and $59(11 \%)$ participants had more than 10 condomless partners where the participant was receptive and 77 (14\%) had more than 10 condomless partners where the participant was insertive.

Participants reported a variety of reasons for not using a condom at their last condomless anal sex act: it is more enjoyable without a condom $(n=349 ; 65 \%)$; I don't like using condoms ( $n=268 ; 50 \%)$; he doesn't like using condoms $(n=178$; $33 \%)$; condoms weren't discussed $(n=140 ; 26 \%)$; I was under the influence of drugs $(n=128 ; 24 \%)$ or alcohol $(n=113 ; 21 \%)$; I didn't consider myself at risk of $\operatorname{HIV}(n=115 ; 21 \%)$; and we don't use condoms with each other but do with other partners $(n=86 ; 16 \%)$. MCA did not decompose responses to reasons for non-condom use into clear groups. At the last condomless anal sex act 239 (45\%) participants thought their partner was HIV-negative; 146 (28\%) thought their partner was HIV-positive and on treatment; 118 (22\%) did not know the HIV status of their partner; and 28 (5\%) thought he was HIV-positive and either not on treatment or did not know.

Among the 467 participants who reported sexual behaviour in both the last 30 days and the last 90 days at enrolment, participants typically reported more partners per 30 days in the last 30 days (median $=5$; IQR $=2-10$ ) compared to the last 90 days (median $=3$; IQR $=2-7$; $p<0.001)$. This pattern was also consistent for the number of condomless partners where the participant was receptive $($ median $(\mathrm{IQR})=1(0-3)$ versus $1(0-1)$; 
$p<0.001)$ and insertive (median $(\mathrm{IQR})=1(1-3)$ versus $1(0-2) ; p<0.001)$.

Participants in the PROUD pilot study reported their recreational drug use in the past 3 months: 394 (73\%) had used recreational drugs during this period. Poppers and Viagra were frequently used $(n=262 ; 49 \%$ and $n=$ 223; $41 \%$ respectively). Other drugs used included: mephedrone ( $n=197 ; 36 \%$ ), GHB/GBL (gamma-hydroxybutrate or gamma-butyrolactone) $(n=169 ; 31 \%)$, cocaine $(n=139 ; 26 \%)$, cannabis $(n=128 ; 24 \%)$, crystal methamphetamine $(n=98 ; 18 \%)$, ecstasy $(n=90 ; 17 \%)$, ketamine $(n=89 ; 16 \%)$ or some other drug $(n=92$; $17 \%)$. Drugs commonly associated with drug use in a sexual context (ChemSex: mephedrone, GHB/GBL or crystal methamphetamine) were used by $231 / 525$ (44 \%) participants in the past 3 months.

\section{London centres compared to out-of-London centres}

Participants enrolled in the eight London-based clinics ( $n=375 ; 69 \%)$ were compared to participants enrolled in the five out-of-London clinics $(n=165 ; 31 \%)$ to evaluate differences in baseline demographics, STIs and sexual risk behaviour. Participants in London were less likely to be white ( 75 versus $86 \% ; p=0.003$ ) and more likely to be Black-African, Black-Caribbean or mixed race (10 versus $4 \% ; p=0.023$ ). Participants in London were similar in age (median $=36$ versus 35 years; $p=0.37$ ) to out-of-London participants. London participants were more likely to be born outside the UK (50 versus $18 \%$; $p<0.001)$ and more likely to be university educated (69\% versus $42 \% ; p<0.001$ ). London participants had more HIV and STI tests in the last 12 months (mean of 3.5 versus $2.9 ; p<0.001$ and mean of 3.1 versus $2.7 ; p=$ 0.006 respectively) and more courses of PEP in the past 12 months (mean of 0.64 versus $0.41 ; p=0.022$ ). London participants reported more diagnoses of STIs in the last 12 months than out-of-London participants $(n=243,67 \%$ versus $n=88,56 \% ; p=0.018$ ) and had significantly more lifetime STI diagnoses $(n=342,91 \%$ versus $n=131 ; 80 \%$; $p=0.001$ ). Finally, London participants also reported a higher total number of partners in the last 90 days $($ mean $=17.3$ versus 13.3 ; median $=10$ versus $10 ; \mathrm{IQR}=$ $5-20$ versus $4-18 ; p=0.023)$ and significantly more condomless partners where the participant was insertive (mean of 5.1 versus 3.7 ; median $=2$ versus $1 ; \mathrm{IQR}=$ $1-6$ versus $1-5 ; p=0.029)$. However, there was no significant difference between London and out-of-London participants in the number of condomless partners where the participant was receptive (mean of 4.1 versus 3.7; median $=2$ versus 2 ; IQR $=1-4$ versus $1-4 ; p=$ 0.54 ) or the number of new partners (mean of 11.9 versus 9.3; median $=7$ versus 6 ; IQR $=2-15$ versus $1-11$; $p=0.15)$. London participants were more likely to have used recreational drugs in the past 3 months $(n=287$, $79 \%$ versus $n=107,66 \% ; p=0.003$ ).

\section{GUMCAD clinic comparison}

PROUD participant data were compared to data from 92,307 HIV-negative GMSM who attended a GUMCAD clinic in 2013, of which 40,493 HIV-negative GMSM were seen at participating PROUD clinics [22]. There were significant differences in age between PROUD participants and GUMCAD attendees, with more participants in PROUD aged 34-44 (33\% versus $22 \% ; p<0.001)$ and fewer aged 20-24 (9\% versus $19 \% ; p<0.001)$. The ethnicity of PROUD participants appeared similar to GUMCAD attendees (81\% versus $80 \%$ white ethnicity). PROUD participants were more likely to be born outside the UK compared to GUMCAD attendees (40\% versus $28 \% ; p<0.001)$, but were similar to GUMCAD attendees at PROUD clinics ( $40 \%$ versus $43 \% ; p=0.22$ ). PROUD participants were more likely to have been recruited from London than the general GUMCAD clinic attendees (70 \% versus $51 \% ; p<0.001)$. PROUD study participants had attended clinic a mean of 3.27 times in the past 12 months for an HIV test, compared to a mean of 1.13 for HIV-negative MSM attending GUMCAD clinics in 2013.

Whilst not directly comparable measurements, the number of STI diagnoses reported by PROUD participants is high in comparison to GUMCAD attendees. Rectal chlamydia was diagnosed in 2454 (3 \%) GUMCAD attendees in 2013 and 2244 (2\%) were diagnosed with rectal gonorrhoea. There were a total of 585 primary syphilis diagnoses, 87 hepatitis $\mathrm{C}$ diagnoses and 107 LGV diagnoses in HIV-negative GMSM attending GUMCAD clinics in 2013, all less than $1 \%$. PEP use also appears high among PROUD participants compared to the general GMSM population in England: in GUMCAD clinics in 2013, there was a total of 4133 PEP prescriptions in 92,037 HIV-negative GMSM (4.5 \%).

\section{Discussion}

The PROUD study is the first randomised entirely openlabel study of PrEP with a deferral design that aims to explore its real-world effectiveness, taking account of changes in behaviour that may follow the use of a drug known to reduce the risk of contracting HIV.

The baseline data presented here indicate that the GMSM who enrolled in the PROUD study are at substantially higher risk of acquiring HIV infection sexually than the overall population of GMSM attending sexual health clinics in England. Our data demonstrate that the PROUD cohort had several key indicators to support the observation that they were at high risk of HIV acquisition, particularly those recruited through the London centres. This is consistent with data suggesting that people living 
in London tend to have poorer sexual health outcomes [23, 24], are a population with higher rates of partner change and higher use of ChemSex drugs [25]. These indicators could inform discussions with potential PrEP users in the UK. Further analysis is required to establish whether these indicators are predictive of HIV infection among the participants who seroconverted during the study.

There is a striking excess of reported STIs in the 12 months preceding baseline in the PROUD cohort compared to the number of infections diagnosed in the general HIV-negative GMSM population attending clinics in 2013. This difference is not directly comparable, due to participants' 12-month history of STIs not all occurring in 2013, and because diagnosed STIs are per clinic whilst reported STIs may span several clinics that a participant visits. Nonetheless, this difference suggests that PROUD participants have particularly high rates of STIs. Of note, a high proportion of the PROUD cohort reported previous rectal infections. Rectal infections, particularly with gonorrhoea and chlamydia, have been associated with higher risk of HIV infection and it has been suggested that a diagnosis of rectal STIs may be used as one of a number of criteria for actively recommending PrEP to GMSM clinic attendees [26]. Despite this excess of STIs at baseline, only $22 \%$ of participants considered themselves to be at high or very high risk of HIV in general when they last had anal sex without using a condom. This highlights a limitation of this question as perceived HIV risk at the last anal sex act without a condom will vary by partner and may differ from a participant's overall perceived risk of acquiring HIV.

Participants also reported high numbers of sexual partners, particularly condomless sex partners. The PROUD cohort reported a higher median number of partners in the preceding 90 days, compared to that reported elsewhere. For example, PROUD participants reported 10 partners versus 5 in the European EMIS survey of MSM [27] and versus 3 in the US National Behavioural Surveillance system survey [28]. In the Pre-exposure Prophylaxis Initiative (iPrEx) study, eligible GMSM reported an average of 7 sexual partners in the past 3 months at baseline and an average of 18 sexual partners in the past 12 months [2]. The median number of condomless sex partners reported in PROUD appears high in relation to European and US surveys. In EMIS, 58 \% of GMSM reported at least one instance of condomless anal sex in the past 12 months [27]. In a US survey, white and black GMSM reported a median of two condomless anal sex partners in the past 12 months [29]. Further work should compare the distribution of the number of partners since the median is of limited value.

There is concern about the role of recreational drugs in facilitating condomless anal sex, leading to an increased number of partners and the possibility of rectal trauma after protracted periods of sexual activity. A survey of ChemSex (sex under the influence of drugs) in south London found that a third of men reported difficulty in negotiating safe sex whilst under the influence of drugs [25]. Although PROUD participants were not asked specifically about ChemSex, their use of recreational drugs in the past 3 months, particularly drugs associated with ChemSex such as mephedrone, crystal methamphetamine and GHB/GBL, was much higher than previously reported in national Internet surveys of GMSM [30] and the general population in England and Wales [31].

The study population reported an eight-fold higher use of PEP for sexual exposure in the past year when compared to the national surveillance data, with $13 \%$ using it more than once. The costs associated with PEP are substantial and include drug costs and frequent clinic visits. Cost-effectiveness analyses need to be conducted to determine if a PrEP programme could reduce costs compared to PEP in a population with this level of PEP usage.

PROUD participants used several other risk reduction strategies such as condoms, serosorting and seropositioning. However, despite active risk management, including using strategic positioning, three participants had a reactive HIV antigen-antibody test at baseline, despite a non-reactive antibody point-of-care test. These recently acquired infections underscore the failure of existing risk reduction strategies to prevent infection. This is not surprising as the range of risk reduction strategies employed prior to enrolment were selective, either selective condom use or selection of partner and type of sex to have without a condom. Follow-up data from PROUD will provide an opportunity to assess how men fit PrEP into their existing strategies.

Previous cost-effectiveness studies for PrEP suggested that with current drug costs a daily PrEP programme will only be of cost-benefit if provided to the population most at risk of HIV [32-35]. Data from PROUD will inform decisions about commissioning of PrEP in England [36] and UK cost-effectiveness analyses [37, 38]; these concluded that daily PrEP, in a medium-sized PrEP rollout of 5000 GMSM, would only become cost-effective if current drug prices are substantially reduced. The baseline data suggest that it is feasible to deliver an effective PrEP programme through the sexual health clinic network. PROUD was a pilot study with very limited resources and recruitment depended not only on clinical providers identifying GMSM who would benefit from PrEP, but also GMSM recognising their need for additional options and informing others in their social and sexual networks.

There are some limitations to this analysis. Although data were available for the majority of participants at 
baseline, a small proportion of baseline data were missing. Efforts were made to contact all of these participants to collect basic demographic data. However, we did not retrospectively collect behavioural data as this would have been subject to recall bias. Recall bias may also explain the difference in 90-day versus 30-day risk assessment findings, with the suggestion that 90-day risk assessment may underestimate more recent risk.

The PROUD population were highly educated; they were older and more likely to have been born outside the UK compared to the general GMSM population attending GUM clinics in England, although were as equally likely to have been born outside the UK as attendees from the sexual health clinics in which they were recruited. The PROUD population has a similar median age to the age of first diagnosis among GMSM in England, which was 34 years in 2012 [39]. Due to the time lag between infection and diagnosis, this suggests that GMSM accessing PrEP in PROUD are also older than the age of the general GMSM population who seroconvert.

Black, Asian and minority ethnic (BAME) GMSM populations were similarly represented in the PROUD cohort compared to the population attending the same GUM clinics in England but had higher representation than the general GMSM population in England. Data from the US, UK and Canada suggest that this ethnic group is at highest risk of acquiring HIV and would benefit from PrEP. This needs to be explored further in the UK context where there are fewer disparities in access to health care [40] but where differences in the use of services remain [41].

PrEP was only offered in the PROUD study in major urban centres, and may not have been accessible to those living in more rural areas and in some parts of the country where there were no PROUD centres. In addition, the process of informed consent, randomisation to wait a year, and participation in a study, in general, could have deterred many eligible GMSM from enrolling. This could have contributed to the initial delay in recruitment to the study and forthcoming quantitative and qualitative work will describe the acceptability of the study in more detail. There were also unanticipated delays getting all 13 recruitment centres operational. Nonetheless, the rate of recruitment increased over time as information about the trial spread via word of mouth and the eventual population recruited were at high risk of HIV, so benefited greatly from the offer of PrEP.

Participants who enrolled in the PROUD study knew that PrEP had proven biological efficacy and these results demonstrate that the population interested in PrEP are likely to be highly self-selective for those at high risk of HIV acquisition. We expect similar effectiveness in populations with a lower risk of sexually acquiring HIV if there are similar levels of adherence, although our findings suggest that populations at low risk of HIV or with low rates of STIs may be less interested in the offer of PrEP if it becomes accessible as part of the NHS. Nonetheless, there were some participants in PROUD who had never reported an STI during their lifetime and further research is ongoing examining any changes in sexual risk behavior and STI diagnosis in this group.

\section{Conclusion}

Baseline data from the PROUD pilot study suggest that it attracted a population of GMSM at high risk of HIV, reflected in high rates of STIs and higher risk sexual behaviours. These findings contribute to explaining the extraordinary HIV incidence rate during follow-up. Despite broad eligibility criteria, the population interested in PrEP were highly selective and substantially benefited from access to PrEP.

\section{Additional files}

Additional file 1: Baseline Sexual Behaviour Questionnaire. (DOC 155 kb)

Additional file 2: Adherence and Sexual Behaviour Questionnaire. (DOC $95 \mathrm{~kb}$ )

\section{Abbreviations}

ASTRA: Antiretrovirals, Sexual Transmission Risk and Attitudes; BAME: Black, Asian and minority ethnic; ChemSex: the use of crystal methamphetamine, $\mathrm{GHB} / \mathrm{GBL}$ or mephedrone either immediately before, or during sex; FTC: emtricitabine; GBL: gamma-butyrolactone; GHB: gamma-hydroxybutrate; GMSM: gay and other men who have sex with men; GUM: genitourinary medicine; GUMCAD: genitourinary medicine clinic activity database;

HIV: human immunodeficiency virus; iPrEx: Pre-exposure Prophylaxis Initiative; IQR: interquartile range; LGV: lymphogranuloma venereum; MCA: multiple correspondence analysis; PEP: post-exposure prophylaxis; PrEP: pre-exposure prophylaxis; STI: sexually transmitted infection; TDF: tenofovir; UK: United Kingdom; US: United States.

\section{Competing interests}

The PROUD study was provided drug free of charge by Gilead Sciences plc which also distributed it to participating clinics and provided funds for additional diagnostics which included the pharmacokinetic sub-study.

\section{Authors' contributions}

SM led the study. DID, MD, MG, DTD, ONG, AN and SM conceived and designed the study. AM, RG, AC, MF, GS, AS, NM, IR, MP, JS, JF, JB, MB, CB, CL, ST, DW, SA and SM recruited patients and collected data. DID and MD led on writing this paper and DID conducted the analyses. All authors read and approved the final manuscript.

\section{Acknowledgements}

Barts: Vanessa Apea, Drew Clark, Paul Davis, James Hand, Machel Hunt, Rebecca Neale, Jackie O'Connell, Margaret Portman, Liat Sarner, John Saunders, Louise Terry, Angelina Twumasi, Salina Tsui, Dayan Vijeratnam, Ryan Whyte, Andy Williams

Birmingham: Sian Gately, Gerry Gilleran, Jill Lyons, Chris McCormack, Katy Moore, Cathy Stretton, Stephen Taylor, David White

Brighton: Alex Acheampong, Michael Bramley, Marion Campbell, Ruby Chowdhry, Amanda Clarke, Stewart Eastwood, Babs Fennell, Martin Fisher, Wendy Hadley, Kerry Hobbs, Sarah Kirk, Nicky Perry, Charlotte Rawlinson, Celia Richardson, Claire Richardson, Mark Roche, Emma Simpkin, Simon Shaw, Elisa Souto, Julia Williams, Elaney Youssef

Chelsea and Westminster: Simone Antonucci, Tristan Barber, Cindy Eliot, Serge Fedele, Chris Higgs, Kathryn McCormick, Sheena McCormack, Alan McOwan, 
Alexandra Meijer, Sam Pepper, Jane Rowlands, Gurmit Singh, Alfredo SolerCarracedo, Sonali Sonecha, Ann Sullivan, David Taylor, Lervina Thomas Gilead Sciences: Rich Clarke, Jim Rooney

Homerton: Frederick Attakora, Marina Bourke, Richard Castles, Rebecca Clark Anke De-Masi, Veronica Espa, Rumbidzai Hungwe, Martin Lincoln, Sifiso Mguni, Rhianon Nevin-Dolan, lain Reeves

King's: Hannah Alexander, Jake Bayley, Michael Brady, Lucy Campbell, Sophie Candfield, Shema Doshi, Olivia Liddle, Larissa Mulka, Priyanka Saigal, James Stevenson

Manchester: James Boateng, Brynn Chappell, Susanna Currie, Carolyn Davies, Dornubari Lebari, Matthew Phillips, Gabriel Schembri, Lisa Southon, Sarah Thorpe, Anna Vas, Chris Ward, Claire Warren, Stephanie Yau

Mortimer Market: Alejandro Arenas-Pinto, Asma Ashraf, Matthew Bolton, Richard Gilson, Lewis Haddow, Sara McNamara, Ana Milinkovic, June Minton, Dianne Morris, Clare Oakland, Steve O'Farrell, Pierre Pellegrino, Sarah Pett, Nina Vora, Carmel Young, Taris Zarko-Flynn

MRC CTU at UCL: Sarah Banbury, Elizabeth Brodnicki, Christina Chung, David Dolling, David Dunn, Keith Fairbrother, Mitzy Gafos, Sajad Khan Shabana Khan, Sheena McCormack, Brendan Mauger, Yinka Sowunmi, Susan Spencer, Ellen White, Gemma Wood

Public Health England: Monica Desai, Sarika Desai, Nigel Field, Noel Gill, Kate Hyland, Anthony Nardone, Parnam Seyan

Sheffield: Anthony Bains, Gill Bell, Christine Bowman, Terry Cox, Claire Dewsnap, Charlie Hughes, Hannah Loftus, Naomi Sutton, Debbie Talbot, Vince Tucker Social Science Team: Gill Bell, Caroline Rae, Michael Rayment, Will Nutland, Sonali Wayal

St Marys: Wilbert Ayap, Ling Jun Chen, Adam Croucher, Sarah Fidler, Kristin Kuldanek, Ken Legg, Agathe Leon, Nicola Mackie, Nadia Naous, Killian Quinn, Severine Rey, Judith Zhou

St Thomas': Margaret-Anne Bevan, Julie Fox, Nina Francia, Eleanor Hamlyn, Lisa Hurley, Helen Iveson, Isabelle Jendrulek, Tammy Murray, Alice Sharp, Andrew Skingsley, Chi Kai Tam, Al Teague, Caroline Thomas, Juan-Manuel Tirabosch

York: Christine Brewer, Richard Evans, Jan Gravely, Charles Lacey, Gary Lamont, Fabiola Martin, Georgina Morris, Sarah Russell-Sharpe, John Wightman The PROUD Study Group

\section{Funding}

The study was supported by ad hoc funding from the MRC Clinical Trials Unit at University College London and an innovations grant from Public Health England, and most clinics received support through the UK National Institute of Health Research Clinical Research Network. Gilead Sciences provided Truvada, distributed drug to clinics, and awarded a grant for the additional diagnostic tests including drug concentrations in plasma.

\section{Author details}

${ }^{1}$ MRC Clinical Trials Unit at UCL, Aviation House, 125 Kingsway, London WC2B 6NH, UK. ${ }^{2} \mathrm{HIV} / \mathrm{STI}$ Department, Public Health England, London, UK. ${ }^{3}$ Chelsea and Westminster Hospital NHS Foundation Trust, London, UK. ${ }^{4}$ The Mortimer Market Centre, Central and Northwest London NHS Foundation Trust, London, UK. ${ }^{5}$ Claude Nichol Centre, Royal Surrey Sussex County Hospital, Brighton, UK. ${ }^{6}$ Manchester Centre for Sexual Health, Central Manchester University Hospitals NHS Foundation Trust, Manchester, UK. ${ }^{7}$ St Mary's Hospital, Imperial College NHS Foundation Trust, London, UK. ${ }^{8}$ Homerton University Hospital NHS Foundation Trust, London, UK. ${ }^{9}$ Ambrose King Centre, Barts Health NHS Trust, London, UK. ${ }^{10}$ Guy's and St Thomas' NHS Foundation Trust, London, UK. ${ }^{11}$ King's College Hospital NHS Foundation Trust, London, UK. ${ }^{12}$ Sheffield Teaching Hospitals NHS Foundation Trust, Sheffield, UK. ${ }^{13}$ York Hospitals NHS Foundation Trust, York, UK. ${ }^{14}$ Heart of England NHS Foundation Trust, Birmingham, UK.

\section{Received: 28 April 2015 Accepted: 14 March 2016}

Published online: 24 March 2016

\section{References}

1. Holmes D. FDA paves the way for pre-exposure HIV prophylaxis. Lancet. 2012:380(9839):325.

2. Grant RM, Lama JR, Anderson PL, McMahan V, Liu AY, Vargas L, et al. Preexposure chemoprophylaxis for HIV prevention in men who have sex with men. N Engl J Med. 2010;363(27):2587-99. doi:10.1056/NEJMoa1011205.
3. Baeten JM, Donnell D, Ndase P, Mugo NR, Campbell JD, Wangisi J, et al. Antiretroviral prophylaxis for HIV prevention in heterosexual men and women. N Engl J Med. 2012;367(5):399-410. doi:10.1056/NEJMoa1108524.

4. Thigpen MC, Kebaabetswe PM, Paxton LA, Smith DK, Rose CE, Segolodi TM, et al. Antiretroviral preexposure prophylaxis for heterosexual HIV transmission in Botswana. N Engl J Med. 2012;367(5):423-34 doi:10.1056/NEJMoa1110711.

5. Yin ZBA, Hughes G, Nardone A, Gill ON, Delpech VC and contributors. HIV in the United Kingdom 2014 Report: data to end 2013. In: Public Health England. London: PHE publications; 2014

6. Desai M, Desai S, Nardone A, McCormack S, Gill ON. Estimating the impact of a PrEP programme in England for MSM. London: IAPAC: controlling the HIV epidemic with antiretorivirals; 2014.

7. Gomez GB, Borquez A, Case KK, Wheelock A, Vassall A, Hankins C. The cost and impact of scaling up pre-exposure prophylaxis for HIV prevention: a systematic review of cost-effectiveness modelling studies. PLoS Med. 2013; 10(3):e1001401. doi:10.1371/journal.pmed.1001401.

8. Desai M, Gafos M, Dolling D, McCormack S, Nardone A, PROUD study. Healthcare providers' knowledge of, attitudes to and practice of preexposure prophylaxis for HIV infection. HIV Med. 2016;17(2):133-42. http:// dx.doi.org/10.1111/hiv.12285.

9. van der Straten A, Van Damme L, Haberer JE, Bangsberg DR. Unraveling the divergent results of pre-exposure prophylaxis trials for HIV prevention. AIDS. 2012;26(7):F13-9. doi:10.1097/QAD.0b013e3283522272.

10. Golub SA, Kowalczyk W, Weinberger CL, Parsons JT. Preexposure prophylaxis and predicted condom use among high-risk men who have sex with men. J Acquir Immune Defic Syndr. 2010;54(5):548-55. doi:10.1097/QAl. 0b013e3181e19a54.

11. Hurt CB, Eron Jr JJ, Cohen MS. Pre-exposure prophylaxis and antiretroviral resistance: HIV prevention at a cost? Clin Infect Dis. 2011:53(12):1265-70. doi:10.1093/cid/cir684

12. Horberg $M$, Raymond B. Financial policy issues for HIV pre-exposure prophylaxis: cost and access to insurance. Am J Prev Med. 2013;44 1 Suppl 2:S125-8. doi:10.1016/j.amepre.2012.09.039.

13. Foreman C, Gazzard B, Johnson M, Sharott P, Collins S. Maintaining cost-effective access to antiretroviral drug therapy through a collaborative approach to drug procurement, consensus treatment guidelines and regular audit: the experience of London HIV commissioners and providers. Sex Transm Infect. 2012;88(2):112-5. doi:10.1136/sextrans2011-050438.

14. McCormack S, Dunn DT, Desai M, Dolling DI, Gafos M, Gilson R, et al. Pre-exposure prophylaxis to prevent the acquisition of HIV-1 infection (PROUD): effectiveness results from the pilot phase of a pragmatic openlabel randomised trial. Lancet. 2015. doi:10.1016/s0140-6736(15)00056-2

15. Kroenke K, Spitzer RL, Williams JBW. The PHQ-9. J Gen Intern Med. 2001; 16(9):606-13. doi:10.1046/j.1525-1497.2001.016009606.x.

16. Genitourinary medicine clinic activity dataset (GUMCADv2) [database on the Internet]. 2013. Available from: https://www.gov.uk/genitourinary-medicineclinic-activity-dataset-gumcadv2. Accessed 22 March 2016.

17. StataCorp. Stata Statistical Software: Release 13. College Station, TX: StataCorp LP; 2013.

18. De Leeuw J, Mair P. Gifi Methods for Optimal Scaling in R: The Package homals. Journal of Statistical Software. 2009;31(4). doi:10.18637/jss.v031.i04.

19. Dolling D, Desai M, Apea V, Mackie N, McOwan A, Youssef E, et al. Who accesses PrEP? An analysis of baseline data in the PROUD pilot. Liverpool: Third Joint Conference of BHIVA with BASHH; 2014.

20. Lampe F SA, Phillips A, Sherr L, Gilson R, Johnson M, Fisher M, Anderson J, Wilkins E, Broussard C, McDonnell J, Perry N, Scourse R, George V, Hart G, Johnson A, Collins S, Miners A, Elford J, Geretti A, Burman B, Rodger A, for the ASTRA Study. Depression and virological status among UK HIV outpatients: a multicentre study. British HIV Association conference 2012; 18-20 April 2012; 2012.

21. Martin A, Rief W, Klaiberg A, Braehler E. Validity of the Brief Patient Health Questionnaire Mood Scale (PHQ-9) in the general population. Gen Hosp Psychiatry. 2006;28(1):71-7. doi:10.1016/j.genhosppsych.2005.07.003.

22. Genitourinary Medicine Clinic Activity Dataset (GUMCAD). Demographic and STI data for HIV negative MSM attending PROUD clinics: Data sourced from the Genitourinary Medicines Clinic Activity Dataset (GUMCAD) v 2 submitted to Public Health England (PHE) 20132014.

23. Public Health England. STI data tables for England 2013. Table 5: all STI diagnoses and services by gender and sexual risk. 2009 to 2013. In: Public Health England. 2014 
24. London Knowledge and Intelligence Team at Public Health England. Sexual Health. 2012. http://www.lho.org.uk/LHO_Topics/Health_Topics/Lifestyle_ and_Behaviour/SexualHealth.aspx. Accessed 22 March 2016.

25. Bourne ARD, Hickson F, Rueda ST, Weatherburn P. The Chemsex Study: drug use in sexual settings among gay and bisexual men in Lambeth, Southwark and Lewisham London School of Hygiene \& Tropical Medicine. 2014.

26. Pathela P, Braunstein SL, Blank S, Schillinger JA. HIV incidence among men with and those without sexually transmitted rectal infections: estimates from matching against an HIV case registry. Clin Infect Dis. 2013;57(8):1203-9. doi:10.1093/cid/cit437.

27. The EMIS Network. EMIS 2010: The European Men-Who-Have-Sex-With-Men Internet Survey. Findings from 38 countries. Stockholm: European Centre for Disease Prevention and Control; 2013.

28. Rosenberg ES, Sullivan PS, Dinenno EA, Salazar LF, Sanchez TH. Number of casual male sexual partners and associated factors among men who have sex with men: results from the National HIV Behavioral Surveillance system. BMC Public Health. 2011;11:189. doi:10.1186/1471-2458-11-189.

29. Rosenberg E, Kelley C, O'Hara B, Frew P, Peterson T, Sanchez C, et al. Equal behaviors, unequal risks: the role of partner transmission potential in racial HIV disparities among men who have sex with men (MSM) in the US. Washington D.C: 19th International AIDS Conference; 2012.

30. Hickson F, Weatherburn P, Reid D, Jessup K, Hammond G. Consuming passions: findings from the United Kingdom Gay Men's Sex Survey 2005. London: Sigma Research; 2007

31. Home Office. Drug misuse declared: findings from the 2011/12 Crime Survey for England and Wales. 2012.

32. Paltiel AD, Freedberg KA, Scott CA, Schackman BR, Losina E, Wang B, et al. HIV preexposure prophylaxis in the United States: impact on lifetime infection risk, clinical outcomes, and cost-effectiveness. Clin Infect Dis. 2009; 48(6):806-15. http://dx.doi.org/10.1086/597095. Accessed 22 March 2016.

33. Gomez GB, Borquez A, Caceres CF, Segura ER, Grant RM, Garnett GP, et al. The potential impact of pre-exposure prophylaxis for HIV prevention among men who have sex with men and transwomen in Lima, Peru: a mathematical modelling study. PLoS Med. 2012;9(10):e1001323. doi:10.1371/journal.pmed.1001323.

34. Juusola JL, Brandeau ML, Owens DK, Bendavid E. The cost-effectiveness of preexposure prophylaxis for HIV prevention in the United States in men who have sex with men. Ann Intern Med. 2012;156(8):541-50. doi:10.7326/0003-4819-156-8-201204170-00001.

35. Schneider K, Gray RT, Wilson DP. A cost-effectiveness analysis of HIV preexposure prophylaxis for men who have sex with men in Australia. Clin Infect Dis. 2014;58(7):1027-34. doi:10.1093/cid/cit946.

36. Palmer JD, Edwards C. Pre-Exposure Prophylaxis (PrEP) to prevent HIV: clarification of commissioning position. In: NHS England. 2015

37. Cambiano V, Miners A, Dunn D, McCormack S, Gill N, Nardone A, et al. Is pre-exposure prophylaxis for HIV prevention cost-effective in men who have sex with men who engage in condomless sex in the UK? Sex Transm Infect. 2015:91 Suppl 1:A1-A. doi:10.1136/sextrans-2015-052126.1.

38. Punyacharoensin N, Edmunds WJ, De Angelis D, Delpech V, Hart G, Elford J, et al. Effect of pre-exposure prophylaxis and combination HIV prevention for men who have sex with men in the UK: a mathematical modelling study. Lancet HIV. 2016. doi:10.1016/s2352-3018(15)00056-9

39. Aghaizu ABA, Nardone A, Gill ON, Delpech VC and contributors. HIV in the United Kingdom 2013 Report: data to end 2012. In: Public Health England. London: PHE publications; 2013

40. Millett GA, Peterson JL, Flores SA, Hart TA, Jeffries WL, Wilson PA, et al. Comparisons of disparities and risks of HIV infection in black and other men who have sex with men in Canada, UK, and USA: a meta-analysis. Lancet. 2012;380(9839):341-8. doi:10.1016/S0140-6736(12)60899-X.

41. Sonnenberg P, Clifton S, Beddows S, Field N, Soldan K, Tanton C, et al. Prevalence, risk factors, and uptake of interventions for sexually transmitted infections in Britain: findings from the National Surveys of Sexual Attitudes and Lifestyles (Natsal). Lancet. 2013;382(9907):1795-806. doi:10.1016/s01406736(13)61947-9.

\section{Submit your next manuscript to BioMed Central and we will help you at every step:}

- We accept pre-submission inquiries

- Our selector tool helps you to find the most relevant journal

- We provide round the clock customer support

- Convenient online submission

- Thorough peer review

- Inclusion in PubMed and all major indexing services

- Maximum visibility for your research

Submit your manuscript at www.biomedcentral.com/submit 